OPEN

SUBJECT AREAS:

$\mathrm{BIOTIC}$

GENETICS

MICROBIAL GENETICS

FUNGAL PATHOGENESIS

Received

20 May 2013

Accepted

23 July 2013

Published

9 August 2013

Correspondence and requests for materials should be addressed to R.A.W. (rwilson10@ unl.edu)

\section{Growth in rice cells requires de novo purine biosynthesis by the blast fungus Magnaporthe oryzae}

\author{
Jessie Fernandez, Kuan Ting Yang, Kathryn M. Cornwell, Janet D. Wright \& Richard A. Wilson
}

Department of Plant Pathology, University of Nebraska-Lincoln, Lincoln, NE 68583, USA.

Increasing incidences of human disease, crop destruction and ecosystem perturbations are attributable to fungi and threaten socioeconomic progress and food security on a global scale. The blast fungus Magnaporthe oryzae is the most devastating pathogen of cultivated rice, but its metabolic requirements in the host are unclear. Here we report that a purine-requiring mutant of $M$. oryzae could develop functional appressoria, penetrate host cells and undergo the morphogenetic transition to elaborate bulbous invasive hyphae from primary hyphae, but further in planta growth was aborted. Invasive hyphal growth following rice cell ingress is thus dependent on de novo purine biosynthesis by the pathogen and, moreover, plant sources of purines are neither available to the mutant nor required by the wild type during the early biotrophic phase of infection. This work provides new knowledge about the metabolic interface between fungus and host that might be applicable to other important intracellular fungal pathogens.

ice (Oryza sativa) provides $23 \%$ of the calories consumed by humankind, but harvests are frequently lost to the blast fungus Magnaporthe oryzae ${ }^{1}$. Rice blast disease is a severe threat to food global security ${ }^{2-4}$, prompting the development of $M$. oryzae as a model phytopathogen ${ }^{5}$. Plant infection starts when a three-celled asexual spore of $M$. oryzae lands on the surface of a rice leaf and germinates. After a period of polarized growth, the tip of the germ tube begins to swell and form a specialized dome-shaped structure called the appressorium ${ }^{1,6,7}$. Maturation of the appressorium results in the generation of enormous hydrostatic turgor pressure $^{1}$ that acts on a thin penetration hypha, forcing it through the leaf cuticle. In the plant, M. oryzae displays an infection strategy known as a hemibiotrophy such that it colonizes living host cells as a symptomless biotroph for the first 4-6 days of infection before becoming necrotrophic ${ }^{8}$. The biotrophic phase begins when the penetration peg expands to become a thin filamentous primary hypha in the cell lumen before elaborating into bulbous invasive hyphae $(\mathrm{IH})^{3,8}$. The IH pushes into host cells surrounded by the invaginated plant-derived plasma membrane, termed an extra-invasive hyphal membrane (EIHM). After 8 to 12 hours of growth - and following constriction of the $\mathrm{IH}$ - the fungus moves from the first infected cell into neighbouring cells surrounded by the EIHM at points thought to be plasmodesmata ${ }^{8}$. The IH grows as thin primary hyphae in the second set of invaded cells before enlarging again into bulbous IH. Movement to subsequent cells is more rapid, requiring only 2-3 hours of IH growth. A novel structure known as the biotrophic interfacial complex (BIC) is formed in primary hyphae as it enters each cell, and remains behind the IH as it elaborates and grows ${ }^{9-11}$. Secreted effector proteins, some with roles in suppressing plant defenses, accumulate in the BIC. These include the biotrophy-associated secreted (BAS) proteins ${ }^{9}$, the known effectors Avr-Pita, PWL1, PWL2 ${ }^{10}$ and AvrPiz- $\mathrm{t}^{12}$, and the secreted protein MC69. ${ }^{13}$.

Biotrophic growth requires nutrient acquisition. Recently, rice infection has been shown to be dependent on the M. oryzae glucose 6-phosphate (G6P) sensor Tps1, a signaling protein that couples carbon and nitrogen metabolism in response to available glucose ${ }^{3,14-17}$. Loss of Tps1 function abolishes pathogenicity, in part due to the reduced expression of known Tps1-dependent virulence genes ${ }^{15,17}$ and the early expression of genes normally repressed by Tps1 in response to G6P sensing, such as those encoding some cell wall-degrading enzymes ${ }^{17}$. Tps1 thus presents as an important link between primary fungal metabolism - ie. the exploitation of available carbon and nitrogen sources - and the pathogenic process.

Despite elucidating G6P sensing by Tps1 as a central regulator of infection, a significant gap remains in our understanding of the metabolic requirements of $M$. oryzae during biotrophic growth. In particular, how hyphal morphogenesis is influenced and/or controlled by available nutrients in host cells is little understood. The identity and origins (pathogen or host) of important metabolites during infection also remains largely unknown ${ }^{3,18}$. Such 
scarcity of knowledge impedes the development of a robust understanding of the physiology of rice blast disease that would link the cellular descriptions ${ }^{8}$ and genetic dissections ${ }^{17}$ of $M$. oryzae biology and point the way to new, sustainable strategies for combating this problem.

Here, we sought to shed more light on $M$. oryzae metabolic requirements during the biotrophic phase of rice infection by targeting purine metabolism in the fungus. We deleted the SAICAR synthetase-encoding gene MoADE1 to show that de novo purine biosynthesis is not required for appressorium formation or rice cuticle penetration but is essential for establishing biotrophic growth in rice cells. We thus reveal a new metabolic constraint for rice blast disease: the absolute dependency of rice blast disease on fungal purine metabolism. The implications for understanding pathogen nutrient utilization in planta are discussed.

\section{Results}

The M. oryzae SAICAR synthetase orthologue MoADE1 is required for de novo adenine biosynthesis. Guy11, the wild type isolate of the rice blast fungus $M$. oryzae used in this study, synthesizes purines and pyrimidines de novo. Following completion of the $M$. oryzae genome $e^{5}$, putative purine biosynthetic pathways in $M$. oryzae have been delineated in the Kyoto Encyclopedia of Genes and Genomes (KEGG) by homology to purine metabolic genes characterized in other fungi. We sought to disrupt purine biosynthesis in $M$. oryzae by targeting the SAICAR synthetase-encoding gene, named $A D E 1$ in Saccharomyces cerevisiae ${ }^{19}$. Interrogating the M. oryzae genome, we found the locus MGG_12537 encoded a SAICAR synthetase orthologue, and we subsequently deleted the MoADE1 coding region from the $M$. oryzae genome using our highthroughput split marker method ${ }^{15}$. Like $A D E 1$ mutants in yeast,

a
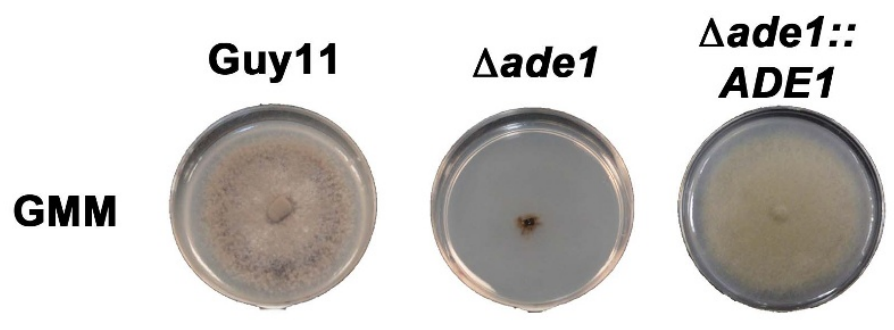

b

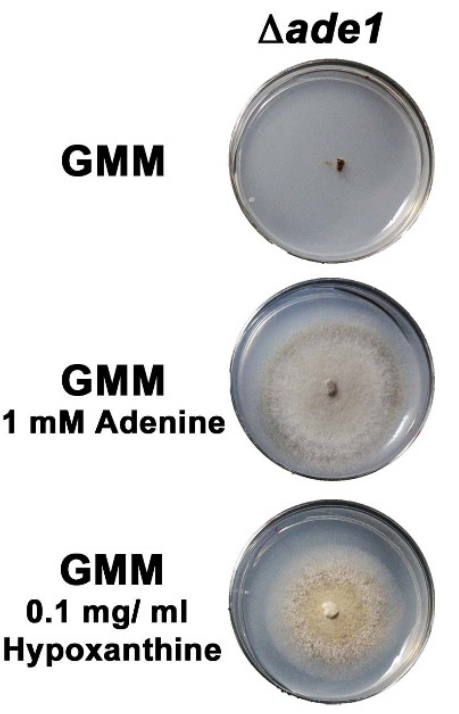

d

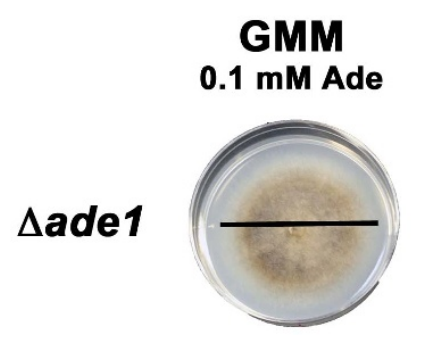

C
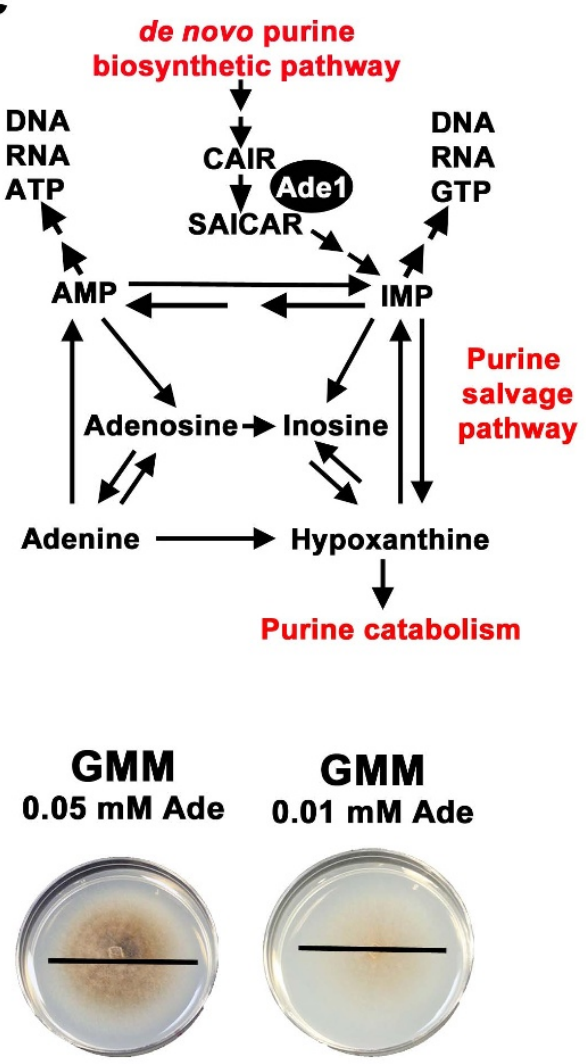

Figure $1 \mid$ Deleting MoADE1, encoding SAICAR synthetase, from the M. oryzae genome generates a conditionally lethal, purine-requiring mutant. (a) $\triangle$ ade1 strains, unlike wild type Guy11 and $\triangle a d e 1$ complementation strains carrying a full-length copy of MoADE1, were unable to grow on glucose minimal media (GMM) lacking an exogenous source of purines such as adenine. (b) Growth of $\triangle$ ade1 strains was restored on GMM supplemented with $1 \mathrm{mM}$ adenine or $0.1 \mathrm{mg} / \mathrm{ml}$ hypoxanthine. (c) Model of purine metabolism in $M$. oryzae, based on KEGG and adapted from ${ }^{31}$, with steps omitted for clarity. Adel functions to convert CAIR to SAICAR in the purine de novo biosynthetic pathway. $\Delta$ adel growth remediation by adenine and hypoxanthine on GMM shows the purine salvage pathway is active in M. oryzae. Hypoxanthine can also be metabolized as a sole nitrogen source by $M$. oryzae ${ }^{14}$, confirming the purine catabolism pathway is also operational in M. oryzae. (d) Adenine added to GMM at the concentrations shown did not affect the radial growth of $\Delta a d e 1$ strains, suggesting adenine uptake from the media is not impaired in this strain. 
the resulting $\Delta a d e 1$ strain could not grow on defined glucose minimal media (GMM) lacking adenine (Fig. 1a). This was solely due to loss of MoAdel function because growth was restored on GMM by re-introducing the wild type copy of MoADE1 into $\Delta a d e 1$ strains (Fig. 1a). Growth of $\Delta a d e 1$ strains on GMM was also restored if $1 \mathrm{mM}$ adenine or, interestingly, $0.1 \mathrm{mg} / \mathrm{ml}$ (final concentration) hypoxanthine was added to GMM (Fig. 1b). Restored growth with both adenine and hypoxanthine supplementation suggests the purine salvage pathway is operational in $M$. oryzae (Fig. 1c). Next, we grew $\Delta$ ade1 on diminishing amounts of adenine and found mycelial radial growth was not reduced at very low concentrations of adenine (ie. $0.01 \mathrm{mM}$ adenine), suggesting adenine uptake from the media is not impaired in adeninerequiring $\Delta$ ade1 strains (Fig. 1d).

$M o A D E 1$ is required for rice leaf pathogenicity but not appressorium formation. We applied spores of wild type Guy11 and $\Delta$ ade 1 strains - harvested from fungal mycelia grown on complete media
(CM) supplemented with $1 \mathrm{mM}$ adenine - to the leaves of whole plants of the susceptible cultivar CO-39. Fig. 2a shows $\Delta a d e 1$ strains were greatly reduced for pathogenicity on rice leaves compared to Guy11, indicating de novo adenine biosynthesis by $M$. oryzae is essential for rice blast disease. Supplementary Figure S1a shows that complementing $\Delta a d e 1$ strains with a wild type copy of $A D E 1$ restored the ability of these strains to infect rice but adding $1 \mathrm{mM}$ adenine to suspensions of $\Delta a d e 1$ spores and applying them to rice leaves did not remediate infection.

Despite being non-pathogenic, Fig. $2 \mathrm{~b}$ shows that $\Delta a d e 1$ strains could form appressoria on the surface of detached rice leaf sheaths that were indistinguishable from Guy11. Fig. $2 c$ shows that the rates of appressorium formation on detached rice leaf sheaths of $\Delta a d e 1$ and Guy11 strains were not significantly different.

$\Delta$ ade1 strains can penetrate rice leaf cuticles at the same rate as Guy1 1 but in planta development is delayed. Next, we used live-cell imaging to determine if the appressoria formed by $\Delta a d e 1$ were

\section{a Guy11 $\Delta$ ade1 b}
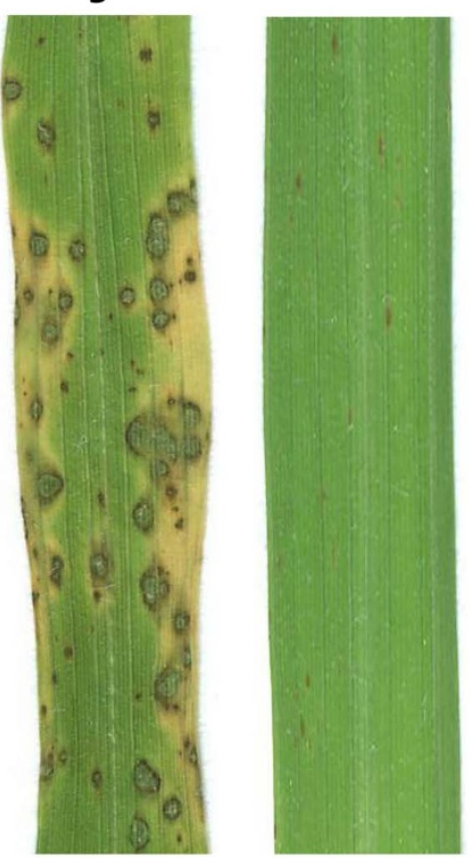

Guy11

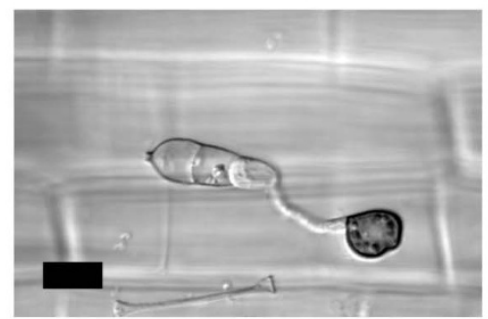

$\Delta$ ade1

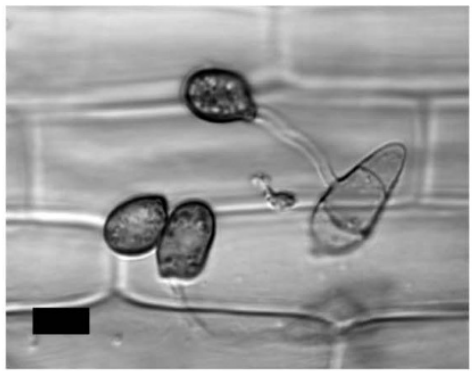

C
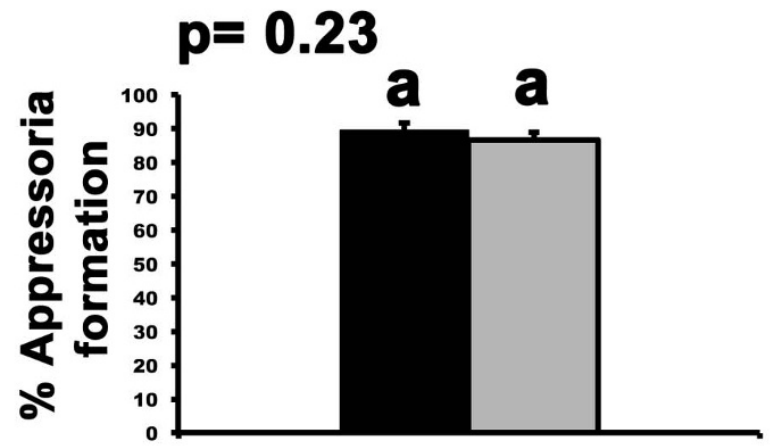

Figure $2 \mid$ Disrupting MoAde1 abolishes infection but not appressorium formation. (a) Spores of $\Delta$ ade1 strains were applied to three-week old plants of the susceptible cultivar CO-39 at a rate of $5 \times 10^{4}$ spores $\mathrm{ml}^{-1}$. Compared to Guy11, $\Delta$ ade1 strains were abolished for pathogenicity. Images were taken after 144 hpi. (b) Despite being non-pathogenic, $\Delta$ ade1 strains were shown to form appressoria on detached leaf surfaces at 24 hpi. Scale bar is $10 \mu \mathrm{m}$. (c) The average rate of appressorium formation on the rice leaf surface was determined. $5 \times 10^{4}$ spores of each strain were inoculated onto rice cuticles. At $24 \mathrm{hpi}$, the number of appressoria that had developed from 50 conidia of each strain was analyzed. This was repeated three times for each strain to determine the mean value. Error bars are standard deviation. Closed bars are values for Guy11 and grey bars are values for $\Delta a d e 1$ strains. Bars with the same letter are not significantly different (Student's $t$-test $\mathrm{p} \leq 0.05$ ). 
functional. Spores of Guy11 and $\Delta a d e 1$ were harvested from fungal mycelia grown on CM plates supplemented with $1 \mathrm{mM}$ adenine, applied to detached rice leaf sheaths, and analyzed for their ability to penetrate rice cuticles at $24 \mathrm{hr}$ post inoculation (hpi). Fig. 3a shows there was no statistical difference (Student's $t$-test $\mathrm{p}=0.21$ ) between the rate of $\Delta a d e 1$ penetration into rice cells compared to Guy11, indicating loss of adenine biosynthesis does not impact the function of $\Delta a d e 1$ appressoria to breach rice cuticles. However, Fig. $3 \mathrm{~b}$ shows that at $24 \mathrm{hpi}$, the subsequent in planta development of $\Delta a d e 1$ strains lagged that of Guy11. By 24 hpi, both Guy11 and $\Delta a d e 1$ strains had formed primary hyphae from the penetration peg, but only those from Guy11 had proceeded to elaborate bulbous IH which, although they had not yet begun to branch, contained a visible BIC structure (Fig. 3b).

IH growth is severely curtailed in $\Delta a d e 1$ strains. Fig. 4a shows that by 40 hpi, Guy11 strains - like $\triangle$ ade1 ADE1 complementation strains (Supplementary Fig. S1b) - had filled the first infected cells and most had spread to adjacent cells. In contrast, $\Delta a d e 1$ strains had, by this time, undergone the morphogenetic transition to elaborate $\mathrm{IH}$ from the primary hyphae, including the formation of BICs, but further growth was attenuated. Supplementary Figure S1b shows that adding $1 \mathrm{mM}$ adenine to spores before applying to detached rice leaf sheaths does not improve the growth of $\Delta a d e 1$ strains in host cells.

By quantifying the differences in IH growth between Guy11 and $\Delta$ ade 1 strains at $40 \mathrm{hpi}$ using the 4-point scale described in a previous study $^{18}$, Fig. $4 \mathrm{~b}$ shows that $\Delta a d e 1$ growth was significantly reduced (Student's t-test $\mathrm{p}=0.0002$ ) in planta compared to Guy11. Fig. 4c shows that by $40 \mathrm{hpi}$, and in contrast to Guy11, no $\Delta a d e 1$ strains had spread from the primary infected cell to adjacent cells. To determine if $\Delta$ ade1 growth rates might change over longer time intervals, Fig. 5 shows that at $75 \mathrm{hpi}$, when Guy11 IH had spread to multiple cells, $\Delta a d e 1$ strains were still confined to the first infected cell. Taken together, these results suggest de novo purine biosynthesis is not required for elaborating appressoria from spores harvested from plates of CM supplemented with $1 \mathrm{mM}$ adenine - nor required for appressorial penetration of the rice cuticle - but is essential for $\mathrm{IH}$ growth in and between host rice cells.

$\Delta$ ade1 strains are alive in host cells at 48 hpi. Plants initiate defense programs in response to pathogen recognition, and these have to be avoided or overcome by the invading pathogen in order for a

\section{a}

$p=0.21$

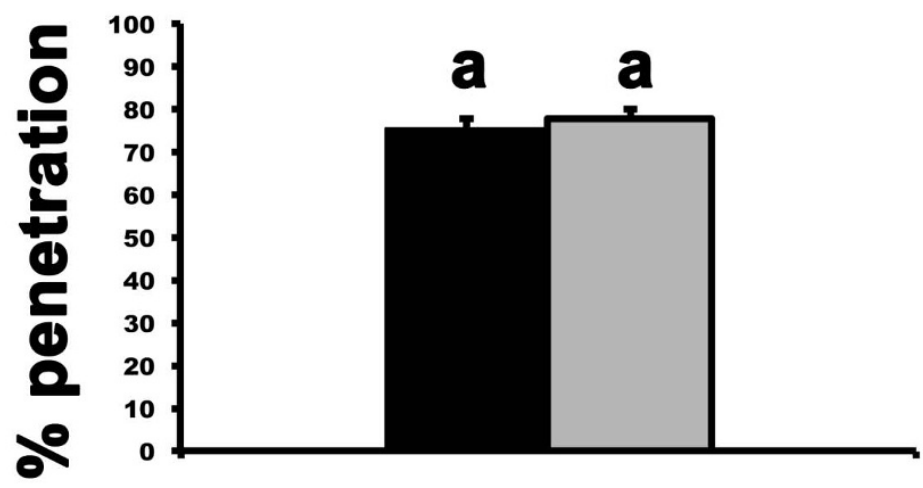

b

\section{Guy11 $24 \mathrm{hpi} \quad \Delta$ ade1}
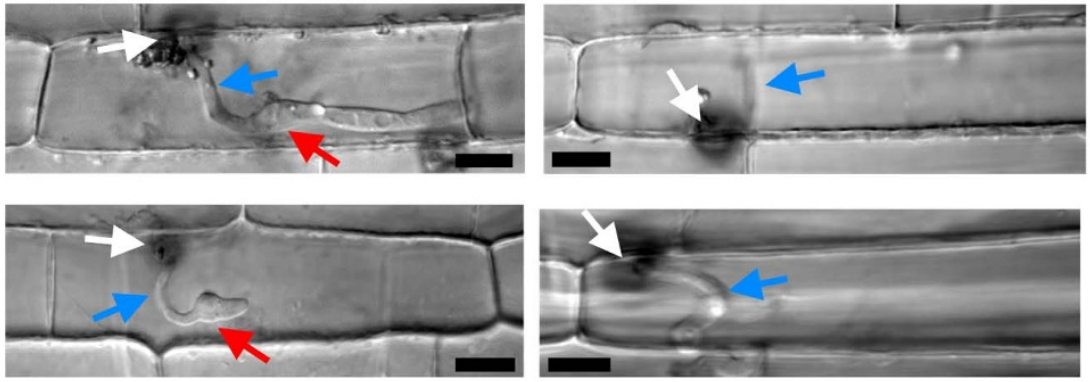

Figure 3 Appressoria of $\mathbf{\Delta a d e 1}$ strains are functional and penetrate leaf cuticles at the same rate as Guy11. (a) The number of appressoria that had penetrated the leaf cuticle, from a total of 50 appressoria observed for each strain, was determined at 24 hpi. The average is calculated from three independent replicates. Error bars are standard deviation. Closed bars are values for Guy11 and grey bars are values for $\Delta a d e 1$ strains. Bars with the same letter are not significantly different (Student's $t$-test $\mathrm{p} \leq 0.05$ ). (b) Representative microscopic observations of fungal development in penetrated leaf epidermal cells at $24 \mathrm{hpi}$. In these examples, Guyl1 appressoria have penetrated the cuticle and formed primary hyphae that have pushed into the epidermal cells and undergone the morphogenetic transition to bulbous IH. BICs have formed between the primary hyphae and IH. In contrast, although $\Delta a d e 1$ appressoria have penetrated rice cuticles at the same rate as Guyl1 and have formed primary hyphae in host cells, elaboration of IH was not observed at $24 \mathrm{hpi}$ in $\Delta a d e 1$ strains. White arrows are appressoria. Blue arrows are primary hyphae. Red arrows are BICs. Scale bar is $5 \mu \mathrm{m}$. 
a

\section{Guy11 \\ 40 hpi}
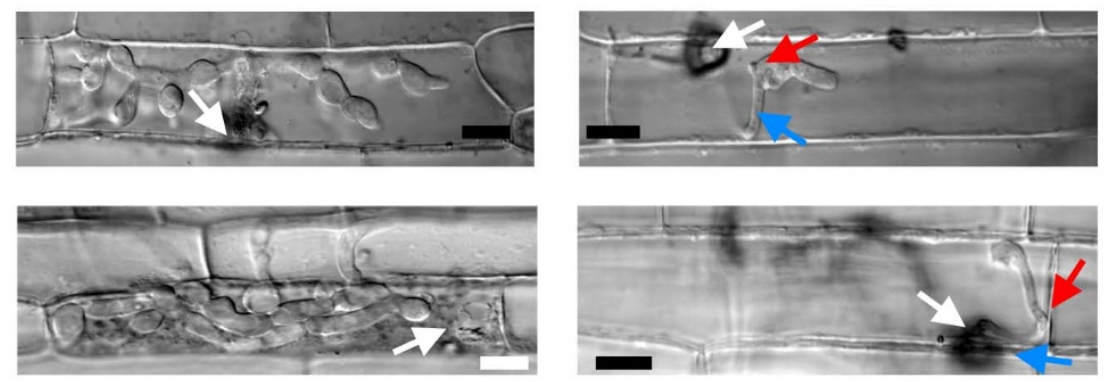

b

$p=0.0002$

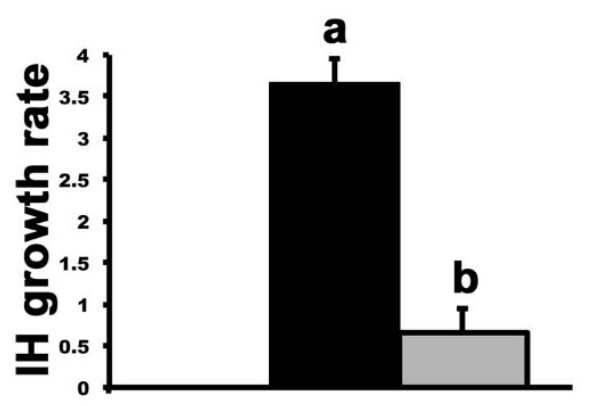

$p \leq 0.0001$

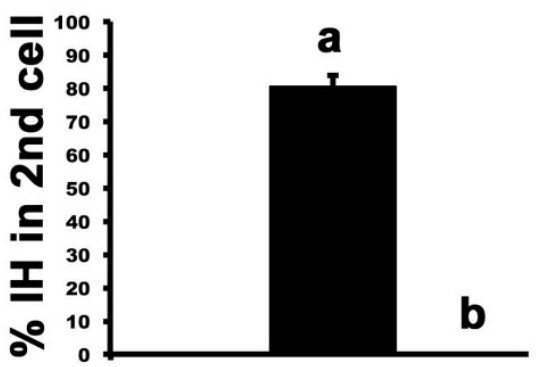

Figure $4 \mid$ Biotrophic growth in rice cells is attenuated in $\Delta$ ade1 strains. (a) At 40 hpi, Guyl1 strains had elaborated branching IH within the first infected cell and were moving to adjacent cells. In contrast, $\Delta a d e 1$ strains, although having undergone the morphogenetic transition to IH from primary hyphae including the formation of BICs, were greatly attenuated in growth. White arrows indicate appressoria and the point of penetration. Blue arrows indicate primary hyphae. Red arrows indicate BICs formed behind IH. Scale bar is $5 \mu \mathrm{m}$. (b) Quantification of the rate of IH growth in rice cells by Guyl1 and $\Delta$ ade1 strains. $\Delta a d e 1$ strains were significantly (Student's $t$-test $\mathrm{p} \leq 0.05$ ) reduced in IH growth compared to Guy 11 . Growth scores were determined using a four-point scale ${ }^{18}$, where $1=\mathrm{IH}$ length shorter than $10 \mu \mathrm{m}$ with no branching; $2=\mathrm{IH}$ length is $10-20 \mu \mathrm{m}$ with $0-2$ branches; $3=\mathrm{IH}$ length is longer than $20 \mu \mathrm{m}$ and/or with more than 2 branches within one cell; $4=\mathrm{IH}$ has spread to adjacent cells. Three leaf sheaths were inoculated with each strain, and IH growth rates were determined for hyphae originating from 50 appressoria per leaf to generate a mean IH growth rate. Error bars are standard deviation. Closed bars are values for Guyl1 and grey bars are values for $\Delta$ ade1strains. Bars with the same letter are not significantly different (Student's t-test $\mathrm{p} \leq 0.05$ ). (c) At $40 \mathrm{hpi}$, no $\Delta$ ade1 hyphae were observed spreading into adjacent secondary cells from primary infected cells. In contrast, approx. $75 \%$ of Guy11 hyphae had spread from the first infected cell into adjacent cells by 40 hpi. Three leaf sheaths were inoculated with each strain, and the average rate of spread of IH into adjacent secondary cells at 40 hpi was determined from a total of 50 primary infected cells per replicate. Error bars are standard deviation. Closed bars are values for Guyl1 and grey bars are values for $\Delta$ adel strains. Bars with the same letter are not significantly different (Student's t-test $\mathrm{p} \leq 0.05)$.

compatible interaction - such as the one that occurs between Guy11 and CO-39 - to occur ${ }^{3,20-22}$. We considered that the attenuated growth observed for $\Delta a d e 1$ strains in rice cells (Fig. 4 and Fig. 5) could result from increased susceptibility to host defenses, relative to Guy11, due to the energetic costs of neutralizing plant defenses not being met in purine-requiring mutant strains. To address this, we sought to determine if $\Delta a d e 1$ strains elicited stronger rice defense responses than Guy11 during infection. Two rice pathogenesisrelated (PR) genes, $P B Z 1$ and $P R 1$, have been previously shown to be more highly induced during challenge by a $M$. oryzae mutant defective in neutralizing plant defenses, $\Delta$ des 1 , than by wild type ${ }^{20}$. In our study, Fig. 6a shows that rice $P B Z 1$ and $P R 1$ expression was not elevated during infection by $\triangle a d e 1$ strains compared to Guy 11 at $48 \mathrm{hpi}$, as determined by quantitative real-time PCR (qPCR) analysis. 48 hpi was chosen because Guyl1 spreads into neighboring cells at this time and $\Delta a d e 1$ strains have undergone the morphogenetic transition to $\mathrm{IH}$ formation. Moreover, Chi and associates ${ }^{20}$ had shown that $\triangle$ des 1 elicited an approximately 100 -fold induction of $P B Z 1$ and $P R 1$ gene expression at 48 hpi compared to wild type. In contrast, Fig. 6a shows $\triangle a d e 1$ strains did not elicit more $P B Z 1$ or $P R 1$ induction compared to Guy11, and $P B Z 1$ expression was greatly reduced in rice cells challenged with $\Delta a d e 1$ compared to Guy11. This could indicate either that $\Delta a d e 1$ strains were not defective in neutralizing plant defense responses, or that the plant had successfully eliminated the threat from $\Delta a d e 1$ colonization by 48 hpi resulting in downregulated $P B Z 1$ expression. To distinguish between these two scenarios, we next sought to determine if $\triangle a d e 1$ strains were alive in rice cells at $48 \mathrm{hpi}$. Fig. $6 \mathrm{~b}$ shows that expression of the M. oryzae actin gene MoACT1 (Table 1) was detected by qPCR analysis of cDNAs resulting from rice leaves infected with either Guy11 or $\Delta a d e 1$ strains but not a mock-inoculated control at 48 hpi. The results were normalized against the expression of the rice actin gene OsAct 2 and hence, the reduced expression of MoACT1 in $\Delta a d e 1$ strains relative to Guy11 reflects the reduced hyphal growth of 


\section{Guy11 75 hpi $\quad \Delta$ ade1}
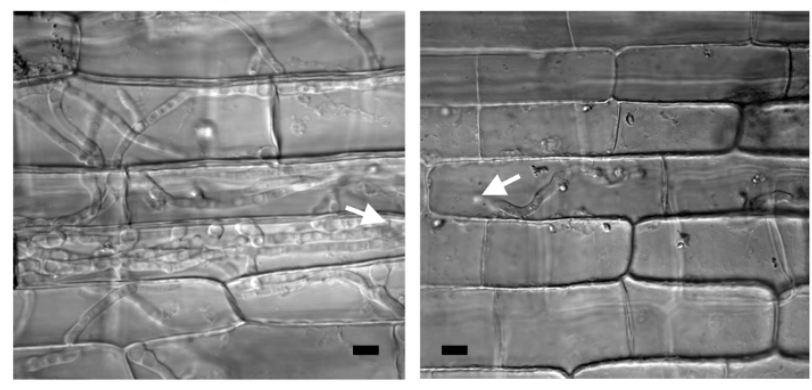

Figure $5 \mid$ No evidence for $\Delta$ ade1 movement beyond the primary infected cell at $75 \mathrm{hpi}$. To determine if $\Delta$ ade 1 strains continue to grow, or if growth accelerates later in infection, we observed Guy11 and $\Delta$ ade1 growth in leaf epidermal cells at 75 hpi. At this timepoint, Guyl1 had spread to multiple adjacent cells, but $\Delta a d e 1$ remained confined to the primary infected cell. White arrows are appressoria and the point of infection. Scale bar is $5 \mu \mathrm{m}$.

this strain. Taken together, these results suggest that reduced growth of $\Delta a d e 1$ strains in rice cells does not occur due to increased susceptibility to rice defenses. Moreover, despite attenuated $\mathrm{IH}$ growth, $\Delta a d e 1$ strains were actively transcribing actin genes in host cells.

Putative nutrient transporters are upregulated in $\Delta a d e 1$ strains at 48 hpi compared to Guy11. On plates, $\Delta a d e 1$ strains can acquire adenine or hypoxanthine from the media (Fig. 1), but our results suggest that in planta, purines in amounts sufficient to remediate IH growth are not available, resulting in the poor growth of $\Delta a d e 1 \mathrm{IH}$ shown in Fig. 4. However, we do not consider this is because rice leaves lack sufficient sources or concentrations of purines, for two reasons. Firstly, we blended $55 \mathrm{~g}$ (wet weight) of 3-week old CO-39 leaves in $50 \mathrm{ml}$ water, removed the plant debris and used the remaining liquid extract to make $100 \mathrm{ml}$ of GMM. Fig. 7a shows that, compared to Fig. 1a, $\Delta a d e 1$ growth was remediated on plates of GMM containing this rice leaf extract. Secondly, Sato and associates $^{23}$ used capillary electrophoresis and mass spectrometry to show that, amongst other metabolites, the purines adenosine,
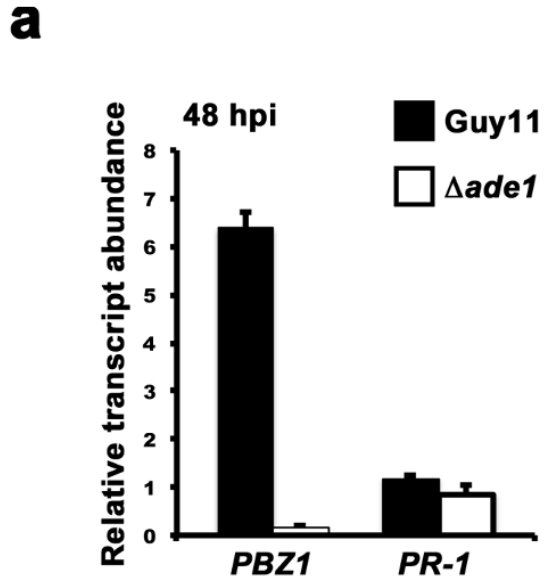

AMP, ADP, ATP, GMP, GDP and GTP were present in measurable quantities in leaf tissue. Concentrations of GMP, GDP and GTP in rice leaves were low $\left(3.6,4.7\right.$ and $4.3 \mathrm{nmol} \mathrm{g}^{-1}$ [fresh weight], respectively) when extracted in the daytime. Concentrations of AMP, ADP and ATP were higher at 89, 60 and

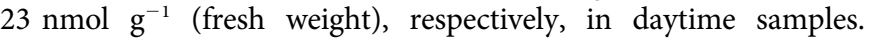
Adenosine concentration was measured at $110 \mathrm{nmol} \mathrm{g}^{-1}$ (fresh weight), ie. $110 \mu \mathrm{M}$, in daytime samples ${ }^{23}$. The concentration of adenine was not determined ${ }^{23}$. Although this study did not specifically measure the concentrations of purines in rice leaf epidermal cells, Borisjuk and colleagues ${ }^{24}$ showed that ATP levels in photosynthesizing cotyledons of faba bean embryos were highest immediately below the surface and were at comparable concentrations $(100 \mu \mathrm{M}-400 \mu \mathrm{M})$ to those measured in rice leaf ${ }^{23}$. It is therefore unlikely rice epidermal cells will be devoid of purines, and so we next sought to determine if the growth of $\Delta a d e 1$ strains could be remediated by utilizing the most abundant purine measured in rice leaf, adenosine, at the concentration determined by Satoh and colleagues ${ }^{23}$. Fig. $7 \mathrm{~b}$ shows that $\Delta a d e 1$ strains could grow on GMM containing decreasing amounts of adenosine including, notably, growth on GMM supplemented with $0.01 \mathrm{mM}$ adenosine, which is 10 -fold less than the concentration of adenosine measured in the rice leaf ${ }^{23}$. Therefore, rice leaf contains compounds that can suppress the purine requirement of $\Delta a d e 1$ on plates (Fig. 7a), and $\Delta$ ade 1 has the ability to acquire small amounts of adenosine from its environment in order to remediate growth (Fig. 7b), but growth does not occur during plant infection (Fig. 5).

To account for the observed deficiency in purine uptake from the plant by $\Delta a d e 1$ strains, we considered that purine transporter gene expression might be repressed in $\Delta a d e 1$ strains, relative to Guy11, during colonization of the host. UAP1 and FCY 2 encode homologues of purine transporters from Aspergillus nidulans ${ }^{25}$ and Saccharomyces cerevisiae ${ }^{26}$, respectively (Table 1 ). Along with the expression of a putative sugar transporter gene unlikely to be involved in purine uptake, RGT2 (Table 1), we determined the expression levels of UAP1 and FCY2 in $\triangle a d e 1$ and Guy11 strains in planta (Fig. 7c). At $48 \mathrm{hpi}$, RGT2 expression was slightly (ie. less than two-fold) reduced in $\triangle a d e 1$ strains compared to Guy11. In contrast, the expression of UAP1 and FCY2 was upregulated approximately 4.5 and 3-fold, respectively, in $\Delta a d e 1$ strains compared to Guy11 at 48 hpi. No cross-hydridization was observed using the M. oryzae gene

b

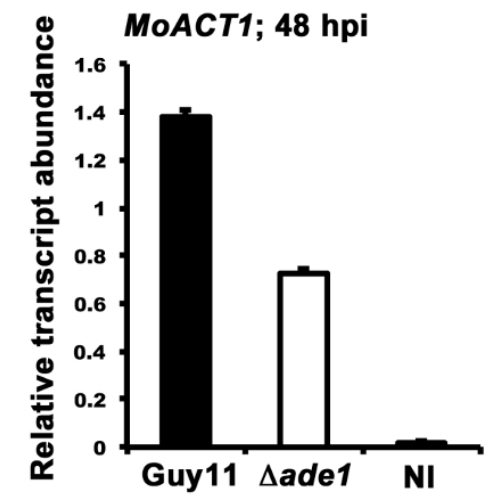

Figure $6 \mid \Delta$ ade1 strains do not elicit strong plant defense responses compared to Guy11. Detached rice leaf sheaths were inoculated with spores of Guy11 or $\triangle a d e 1$ and RNA was harvested at 48 hpi. (a) The expression of the rice pathogenesis-related genes $P B Z 1$ and $P R 1$ was analyzed by qPCR and normalized against the expression of the rice actin-encoding gene OsACT2. $\triangle a$ ade1 did not elicit responses greater than those observed for Guy11, and $P B Z 1$ expression was greatly reduced in rice leaves challenged with $\triangle a d e 1$ strains compared to Guy11. Results are the average of three independent replications. Error bars are standard deviation. (b) M. oryzae actin gene expression was detected in rice leaves challenged with $\Delta a d e 1$ and Guyl1 strains but not an uninfected control (NI), suggesting these strains are actively transcribing in rice cells at 48 hpi. MoAct1 expression was normalized against the expression of the rice actin gene OsACT2. Results are the average of three independent replications. Error bars are standard deviation. 
Table 1 | Description of $M$. oryzae genes analysed by qPCR in this study

\begin{tabular}{|c|c|c|c|}
\hline Locus $^{5}$ & M. oryzae Gene Name & Putative molecular function & Function reference \\
\hline MGG_03982 & MoACT1 & Actin & 5 \\
\hline MGG_08056 & UAPI & $\begin{array}{c}\text { Uric acid-xanthine permease based on similarity to UapA and UapC of Aspergillus } \\
\text { nidulans. }\end{array}$ & 25 \\
\hline MGG_01446 & RGT2 & High affinity glucose transporter & 17,30 \\
\hline
\end{tabular}

specific qPCR primers in uninfected controls (not shown). Thus, although $\Delta a d e 1$ is unable to acquire remediating quantities of purines from the host, this is not likely to be due to the repression of purine transporter genes in this strain.

Taken together, the above results suggest that attenuated $\mathrm{IH}$ growth of $\Delta a d e 1$ strains in rice cells does not likely occur due to an inability to neutralize plant defenses or a reduced capacity to acquire purines compared to Guy11. Moreover, at least up to $48 \mathrm{hpi}, \Delta$ ade 1 strains are actively transcribing genes, including those that might be involved in purine uptake. Regardless, purine acquisition from the host is limiting.

\section{Discussion}

Plant-associated microbes can be beneficial to crops but many are devastating. Sustainable strategies against recalcitrant plant pathogens could come from identifying and exploiting metabolic pathways required for pathogenicity. Here, we characterized the function of $M o A D E 1$, encoding SAICAR synthetase in M. oryzae, in order to progress our understanding of what metabolic processes are intrinsic to the rice blast infection process, and whether the plant host satisfies those requirements. By disrupting MoADE1 function, we have shown that de novo purine biosynthesis (Fig. 8a), but not purine scavenging or uptake from the rice cell (Fig. 8b), is essential for $M$. oryzae growth in planta. This is similar to what has been observed for the human pathogenic fungus Candida albicans, where adenine auxotrophy abolishes virulence in a murine model of systemic candidia$\mathrm{sis}^{27}$, but differs from the obligate biotrophic plant pathogen Blumeria graminis, which diverts purines derived from the host to its own metabolome ${ }^{28}$.

Interestingly, $\Delta a d e 1$ strains could form functional appressoria and elaborate IH from primary hyphae in the first infected rice cells, indicating these initial colonization processes do not require de novo purine biosynthesis (Fig. 8b). Moreover, following the morphogenetic transition to $\mathrm{IH}, \Delta a d e 1$ strains were shown to be alive in host cells at $48 \mathrm{hpi}$ and were actively transcribing actin and transporter genes, and neutralizing host defenses, despite being significantly reduced in growth compared to Guy11. Understanding how IH growth is curtailed, but transcription is permitted, following loss of de novo purine biosynthesis is a future fascinating challenge.

Our results suggest that early biotrophic growth is not dedicated to purine uptake from the host but is instead fuelled by de novo biosynthesis in M. oryzae (Fig. 8a). Additional transcriptional evidence for this strategy is shown in Supplementary Figure 2 where, relative to $R G T 2$, the abundance of UAP1 and FCY2 transcripts were very low during Guy11 infection. This suggests the purine transporters Uap1 and Fcy2 (but not the sugar transporter Rgt2) might not play important roles under normal infection conditions. These observations are consistent with those of O'Connell and associates ${ }^{29}$, who used transcriptional profiling of two hemibiotrophic plant pathogens, Colletotricum graminicola and C. higginsianum, to propose that biotrophic hyphae of $C$. higginsianum function in cell colonization rather than nutrient acquisition. Our results with $M$. oryzae provide the first experimental evidence to suggest that relying on the endogenous production of at least some central metabolites during a

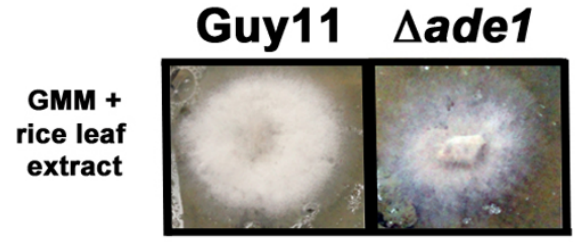

b

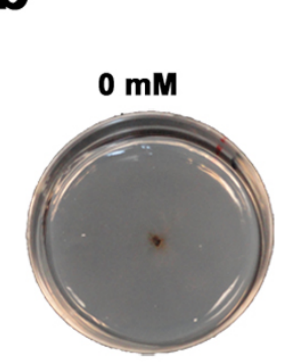

\section{$\Delta$ ade1}
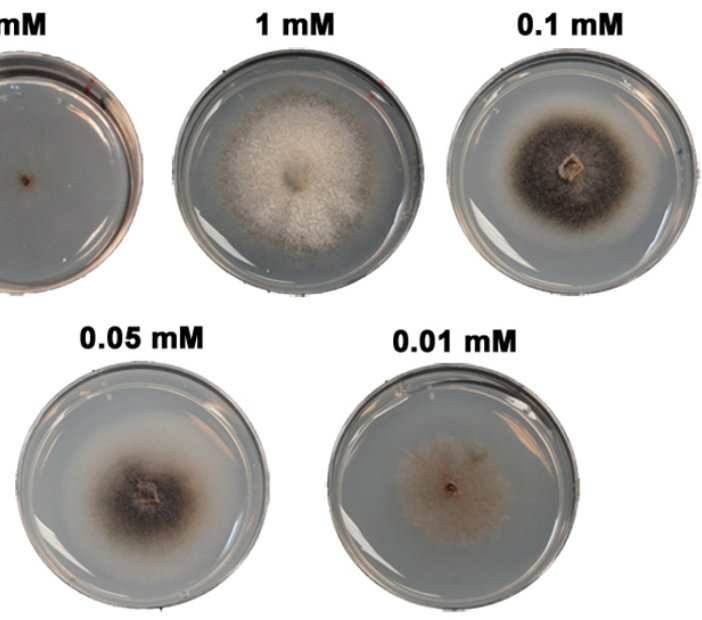

C

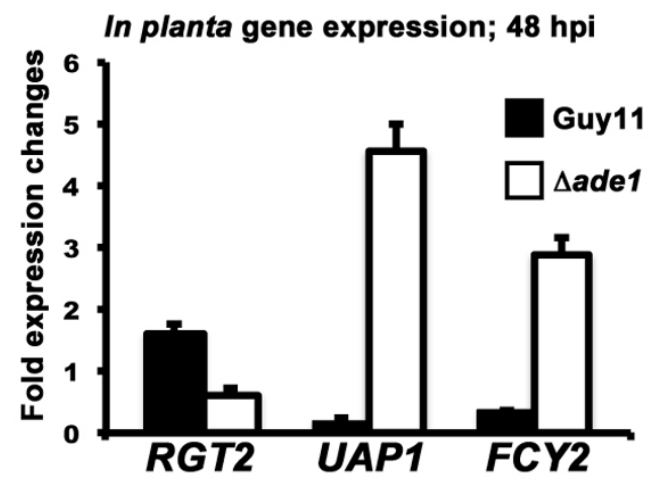

Figure $7 \mid$ Purine uptake from host cells is limiting. (a) $\Delta a d e 1$ strains were able to grow on GMM media containing rice leaf extracts. $55 \mathrm{~g}$ (wet weight) of 3-week old rice leaves were blended in $50 \mathrm{ml}$ of water, pressed through cheesecloth, and the liquid extract used to make $100 \mathrm{ml}$ GMM. (b) $\Delta$ ade1 strains were grown on GMM media with adenosine added at the final concentrations shown. Growth was permissible on GMM supplemented with adenosine at concentrations down to ten-fold less $(0.01 \mathrm{mM})$ than the $110 \mathrm{nmol} \mathrm{g}{ }^{-1}(0.11 \mathrm{mM})$ adenosine found in rice leaves $^{23}$. (c) The expression of two putative purine transporters (UAP1 and FCY2), but not a putative sugar transporter (RGT2), were upregulated 4.5and 3- fold in $\Delta a d e 1$ strains at 48 hpi compared to Guy11. Results were normalized against the expression of the $M$. oryzae actin-encoding gene $M o A C T 1$ and are the average of three independent replications. Error bars are standard deviation. 
a

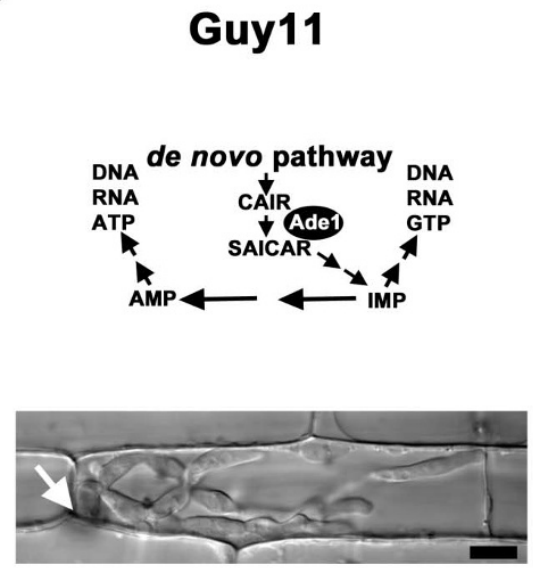

b

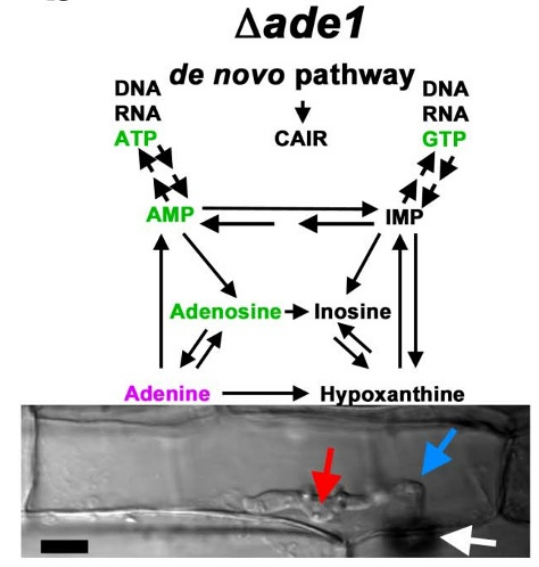

Figure $8 \mid$ de novo purine biosynthesis is essential for growth in rice cells. (a) Model of purine metabolism during biotrophic growth of Guy11 in rice cells (Top), resulting in successful host colonization by $40 \mathrm{hpi}$ (Bottom). (b) Model of purine utilization during biotrophic growth of $\Delta a d e 1$ in rice cells (Top), resulting in attenuated growth at $40 \mathrm{hpi}$ and loss of pathogenicity (Bottom). $\Delta$ adel strains were still alive and transcribing genes at 48 hpi suggesting the recycling of adenine derived initially from the growth media (purple), and/or the limited uptake and salvage of purines known to be in the rice leaf (green), might contribute to survival in the host in the absence of the de novo purine biosynthetic pathway but is not sufficient to drive infection. White arrows indicate appressoria and the point of penetration. Blue arrow indicates primary hyphae. Red arrow indicates BICs formed behind IH. Scale bar is $5 \mu \mathrm{m}$.

biotrophy, rather than acquiring them from the host, might be a common feature of hemibiotrophic pathogens.

What is the advantage to the fungus of having an infection strategy that relies on at least some metabolites being produced endogenously during biotrophy rather than acquired from the host? We do not consider this is because purines, for example, are not present in the plant host at the levels required for growth. Indeed, Sato et al. ${ }^{23}$ have shown that adenosine was present in rice leaves in amounts that, when included in plate tests, were more than sufficient to satisfy the purine requirements for $\Delta a d e 1$ growth (Fig. $7 \mathrm{~b}$ ). Despite this, the failure of $\Delta a d e 1$ strains to grow in planta suggests only limiting amounts of purines, if any, are acquired from host cells (Fig. 8b). Thus, although early biotrophic infection by wild type $M$. oryzae does not require purine acquisition from the plant, $\Delta a d e 1$ strains that require purines are unable to obtain them despite the induction of purine transporter gene expression in planta.

How can we account for the inability of $\Delta a d e 1$ strains to acquire purines from the host, and how does this relate to the requirement for de novo purine biosynthesis during infection? During biotrophic growth, IH is surrounded by the plant-derived EIHM, a structure that the complex molecule FM4-64, a sterol dye, cannot cross ${ }^{8}$. Previously, we have noted that the nutrient utilizing capacity of $M$. oryzae is different in the host compared to axenic growth on media $^{3,18}$. We have suggested one reason for this could be due to the affect of the EIHM on the ability of M. oryzae to acquire nutrients from the host ${ }^{3}$. While glucose acquisition and utilization is a key driver of rice blast disease ${ }^{17}$, the purines discussed in this study, in addition to methionine and aspartate ${ }^{18}$, appear to be limited in availability from the perspective of the fungus during early biotrophic growth. Our data could fit a scenario whereby simple sugars might readily cross the EIHM to be acquired by the fungus from the apoplastic space using, for example, sugar transporters such as Rgt2 (Fig. 7c). On the other hand, more complex molecules such as purines, while abundant in rice leaves (Fig. 7a), might not readily cross the EIHM, resulting in the observed attenuated growth of $\Delta$ ade1 strains. Although in this scenario, maintaining the EIHM inhibits nutrient acquisition and precludes that at least some metabolites must to be produced de novo from endogenous stores, the fungus might retain this strategy if the EIHM confers other growth advantages. Such advantages could include protection from plant defense systems or, as in Colletotricum spp., a platform from which effectors can be delivered into the host cell during biotrophy ${ }^{29}$. Indeed, the $M$. oryzae effectors Slp $1^{22}$ and BAS4 ${ }^{9}$ have been shown to accumulate in the space between the fungal cell wall and the EIHM during biotrophic growth. Thus, the EIHM and the requirement for de novo purine biosynthesis might represent necessary trade-offs in the $M$. oryzae lifestyle between the metabolic demands of the fungus on the one-hand, and effector manipulation of the host cell on the other.

In conclusion, our multi-level approach to understanding nutrient utilization by $M$. oryzae in rice cells - involving molecular genetics, transcriptional analysis and live-cell imaging of infected leaf epidermis - might have signposted the EIHM as a potential mediator of the metabolic interface between pathogen and host. Exploring this role will involve detailed characterizations of the molecular mechanisms by which $M$. oryzae acquires host nutrient across this barrier, characterizing the relationship between nutrient acquisition and the EIHM as biotrophy ends and the fungus becomes necrotrophic, and determining the consequences of perturbing the EIHM during early infection. Such analyses of this metabolic interface might reveal novel pathogenic processes that could be disrupted using chemical or biological means.

\section{Methods}

Fungal manipulations. $\Delta a d e 1$ was derived from Guy $11^{15}$ and is stored as filter stocks at $-20^{\circ} \mathrm{C}$ in the Wilson lab. Strains were maintained on complete medium (CM) containing $1 \%(\mathrm{~W} / \mathrm{V})$ glucose, $0.2 \%(\mathrm{~W} / \mathrm{V})$ peptone, $0.1 \%(\mathrm{~W} / \mathrm{V})$ yeast extract, $0.1 \%$ (W/V) casamino acids and supplemented with $1 \mathrm{mM}$ adenine. Growth studies were performed using Cove's glucose minimal medium $(\mathrm{GMM})^{14}$ with nitrate as sole nitrogen source. Adenine, adenosine or hypoxanthine was added to plates at the concentrations shown. For all plate growth tests, a $5 \mathrm{~mm}$ plug of each strain was transferred from CM to the appropriate media plates and allowed to grow at $26^{\circ} \mathrm{C}$ under $12 \mathrm{hr}$ light/dark cycles for 10 days. $55 \mathrm{~mm}$ petri dishes were used throughout. Plate images were taken with a Sony Cyber-shot digital camera, 14.1 mega pixels.

Targeted gene replacement. Protoplasts were generated and transformed, as described previously ${ }^{15}$. To generate $\Delta a d e 1$ strains, the entire coding region of MoADE1 (MGG_12537) was replaced with the Bar gene conferring bialaphos resistance using the PCR-based split marker method ${ }^{15}$ and the primers shown in Supplementary Table S1. Briefly, $1 \mathrm{~Kb}$ of the left flank (LF) and right flank (RF) of the $M o A D E 1$ coding region were amplified using the primers LF5' \& $\mathrm{LF}^{\prime}$ and RF5' \& RF3', respectively. The 5' region of the Bar gene was amplified using the primer pair M13F:BA and BaSplit, and the $3^{\prime}$ region of the Bar gene was amplified using the primer pair M13R:AR and ArSplit. The MoADE1 left flank amplicon and the $5^{\prime}$ region of the Bar gene were fused by amplifying with NesF and BaSplit. The MoADE1 
right flank amplicon and the $3^{\prime}$ region of the Bar gene were fused by amplifying with NesR and ArSplit. The two resulting fragments, which overlap in the Bar gene by approx. $300 \mathrm{bps}$, were transformed into protoplasts which were initially screened for bialaphos resistance. Targeted gene deletion was confirmed as described in ${ }^{15}$ using LF5' and RF3'. Complementation was achieved by amplifying the full length $M o A D E 1$ gene, including $1 \mathrm{~Kb}$ of flanking sequences, using NesF \& NesR primers, transforming the resulting PCR product into protoplasts of $\Delta a d e 1$ strains, and selecting for restoration of growth on GMM lacking adenine supplementation, as described previously ${ }^{18}$. Complementation strains retained bialaphos resistance.

Plant infection assays and live-cell imaging. Rice plant infections were made using a susceptible dwarf Indica rice (Oryza sativa) cultivar, CO-39, as described previously ${ }^{17}$. Fungal spores were isolated from 12-14 day-old plate cultures of CM supplemented with $1 \mathrm{mM}$ adenine, diluted to a concentration of $1 \times 10^{5}$ spores $\mathrm{ml}^{-1}$ in $0.2 \%$ gelatin and spray-inoculated onto 3-leaf stage rice plants using an artist's airbrush. Disease symptoms were allowed to develop under conditions of high relative humidity for 96 -144 hrs.

Live-cell imaging was performed as described previously ${ }^{18}$ using $3 \mathrm{~cm}$-long leaf sheath segments from 3-4 week-old rice plants and injecting one end of the sheath with a spore suspension of $5 \times 10^{4}$ spores $\mathrm{ml}^{-1}$ in $0.2 \%$ gelatin. At the time points indicated, leaf sheaths were trimmed and observed using a Nikon Eclipse 50i microscope and a Nikon D100 digital net camera.

Physiological studies. The average rates of appressorium formation, penetration and $\mathrm{IH}$ growth were determined for each strain, in triplicate, by analyzing 50 spores or appressoria per rice cuticle. In planta biotrophic growth was determined using the 4point scale described by Wilson and associates ${ }^{18}$, where $1=\mathrm{IH}$ length shorter than $10 \mu \mathrm{m}$ with no branching; $2=\mathrm{IH}$ length is $10-20 \mu \mathrm{m}$ with $0-2$ branches; $3=\mathrm{IH}$ length is longer than $20 \mu \mathrm{m}$ and/or with more than 2 branches within one cell; $4=\mathrm{IH}$ has spread to adjacent cells.

Gene transcript analysis. For $\Delta a d e 1$ and Guy11 in planta gene transcript analysis, rice leaf sheaths were used and RNA was extracted at $48 \mathrm{hpi}$. For transcript analysis of Guyl1 during infection of whole plants, leaves were detached from the plant at the indicated time points, weighed, and approximately $100 \mathrm{mg}$ of tissue was frozen in liquid nitrogen and ground in a mortar and pestle. RNA was extracted from each sample, and uninfected controls, using the RNeasy mini kit from Qiagen. Following treatment with DNase I (Invitrogen), RNA was converted to cDNA using qScript (Quantas). Quantitative real-time PCR (qPCR) was performed on an Eppendorf Mastercycler Realplex using the recommended reagents with primers designed using the netprimer software program (Supplementary Table S1). qPCR data was analyzed using the Realplex software package. Thermocycler conditions were: $10 \mathrm{~min}$ at $95^{\circ} \mathrm{C}$, followed by 40 cycles of $95^{\circ} \mathrm{C}$ for $30 \mathrm{sec}, 63^{\circ} \mathrm{C}$ for $30 \mathrm{sec}$ and $72^{\circ} \mathrm{C}$ for $30 \mathrm{sec}$.

1. Wilson, R. A. \& Talbot, N. J. Under pressure: investigating the biology of plant infection by Magnaporthe oryzae. Nat Rev Microbiol 7, 185-195 (2009).

2. Pennisi, E. Armed and dangerous. Science 327, 804-805 (2010).

3. Fernandez, J. \& Wilson, R. A. Why no feeding frenzy? Mechanisms of nutrient acquisition and utilization during Infection by the rice blast fungus Magnaporthe oryzae. Mol Plant-Microbe Interact 25, 1286-1293 (2012).

4. Fisher, M. C. et al. Emerging fungal threats to animal, plant and ecosystem health. Nature 484, 186-194 (2012).

5. Dean, R. A. et al. The genome sequence of the rice blast fungus Magnaporthe grisea. Nature 434, 980-986 (2005).

6. Dagdas, Y. F. et al. Septin-mediated plant cell invasion by the rice blast fungus, Magnaporthe oryzae. Science 336, 1590-1595 (2012).

7. Ryder, L. S. et al. NADPH oxidases regulate septin-mediated cytoskeletal remodeling during plant infection by the rice blast fungus. Proc Natl Acad Sci USA 110, 3179-3184 (2013)

8. Kankanala, P., Czymmek, K. \& Valent, B. Roles for rice membrane dynamics and plasmodesmata during biotrophic invasion by the blast fungus. Plant Cell 19, 706-724 (2007).

9. Mosquera, G., Giraldo, M. C., Khang, C. H., Coughlan, S. \& Valent, B. Interaction transcriptome analysis identifies Magnaporthe oryzae BAS1-4 as biotrophyassociated secreted proteins in rice blast disease. Plant Cell 21, 1273-1290 (2009).

10. Khang, C. H. et al. Translocation of Magnaporthe oryzae effectors into rice cells and their subsequent cell-to-cell movement. Plant Cell 22, 1388-1403 (2010).

11. Valent, B. \& Khang, C. H. Recent advances in rice blast effector research. Curr Opin Plant Biol 13, 434-441 (2010).

12. Park, C.-H. et al. The Magnaporthe oryzae effector AvrPiz-t targets the RING E3 Ubiquitin Ligase APIP6 to suppress pathogen-associated molecular patterntriggered immunity in rice. Plant Cell 24, 4748-4762 (2012).

13. Saitoh, H. et al. Large-scale gene disruption in Magnaporthe oryzae identifies MC69, a secreted protein required for infection by monocot and dicot fungal pathogens. PLoS Pathog 8, e1002711 (2012).

14. Wilson, R. A. et al. Tps1 regulates the pentose phosphate pathway, nitrogen metabolism and fungal virulence. $E M B O J$ 26, 3673-3685 (2007).
15. Wilson, R. A., Gibson, R. P., Quispe, C. F., Littlechild, J. A. \& Talbot, N. J. An NADPH-dependent genetic switch regulates plant infection by the rice blast fungus. Proc Natl Acad Sci USA 107, 21902-21907 (2010).

16. Fernandez, J. \& Wilson, R. A. The sugar sensor, trehalose-6-phosphate synthase (Tps1), regulates primary and secondary metabolism during infection by the rice blast fungus: Will Magnaporthe oryzae's "sweet tooth" become its "Achilles' heel"? Mycology 2, 46-53 (2011).

17. Fernandez, J. et al. Principles of carbon catabolite repression in the rice blast fungus: Tps1, Nmr1-3, and a MATE-Family Pump regulate glucose metabolism during Infection. PLoS Genet 8, e1002673 (2012).

18. Wilson, R. A. et al. Towards defining nutrient conditions encountered by the rice blast fungus during host infection. PLoS ONE 7, e47392 (2012).

19. Dorfman, B. Z. The isolation of adenylosuccinate synthetase mutants in yeast by selection for constitutive behavior in pigmented strains. Genetics 61, 377-389 (1969).

20. Chi, M. H., Park, S. Y., Kim, S. \& Lee, Y. H. A novel pathogenicity gene is required in the rice blast fungus to suppress the basal defenses of the host. PLoS Pathog $\mathbf{5}$, e1000401 (2009).

21. Huang, K., Czymmek, K. J., Caplan, J. L., Sweigard, J. A. \& Donofrio, N. M. HYR1mediated detoxification of reactive oxygen species is required for full virulence in the rice blast fungus. PLoS Pathog 7, e1001335 (2011).

22. Mentlak, T. A. et al. Effector-mediated suppression of chitin-triggered immunity by Magnaporthe oryzae is necessary for rice blast disease. Plant Cell 24, 322-335 (2012).

23. Sato, S., Soga, T., Nishioka, T. \& Tomita, M. Simultaneous determination of the main metabolites in rice leaves using capillaryelectrophoresis mass spectrometry and capillary electrophoresis diode array detection. Plant J 40, 151-163 (2004)

24. Borisjuk, L. et al. Energy status and its control on embryogenesis of legumes: ATP distribution within Vicia faba embryos is developmentally regulated and correlated with photosynthetic capacity. Plant J 36, 318-329 (2003).

25. Gournas, C., Oestreicher, N., Amillis, S., Diallinas, G. \& Scazzocchio, C. Completing the purine utilisation pathway of Aspergillus nidulans. Fungal Genet Biol 48, 840-848 (2011).

26. Ferreira, T., Chevallier, J., Paumard, P., Napias, C. \& Brèthes, D. Screening of an intragenic second-site suppressor of purine-cytosine permease from Saccharomyces cerevisiae. Possible role of Ser272 in the base translocation process. Eur J Biochem 260, 22-30 (1999).

27. Jiang, L. et al. Functional characterization and virulence study of ADE8 and GUA1 genes involved in the de novo purine biosynthesis in Candida albicans. FEMS Yeast Res 10, 199-208 (2010).

28. Butters, J. A., Burrell, M. M. \& Hollomon, D. W. Purine metabolism in barley powdery mildew and its host. Physiol Plant Pathol 27, 65-74 (1985).

29. O'Connell, R. J. et al. Lifestyle transitions in plant pathogenic Colletotrichum fungi deciphered by genome and transcriptome analyses. Nat Genet 44, 1060-1065 (2012).

30. Ozcan, S., Dover, J., Rosenwald, A. G., Wölfl, S. \& Johnston, M. Two glucose transporters in Saccharomyces cerevisiae are glucose sensors that generate a signal for induction of gene expression. Proc Natl Acad Sci USA 93, 12428-12432 (1996).

31. Oestreicher, N., Ribard, C. \& Scazzocchio, C. The nadA gene of Aspergillus nidulans, encoding adenine deaminase, is subject to a unique regulatory pattern. Fungal Genet Biol 45, 760-775 (2008).

\section{Acknowledgements}

This work was supported by the National Science Foundation (IOS-1145347). Jessie Fernandez was supported by a Hardin Fellowship and a Graduate Student Assistantship from the Department of Plant Pathology, University of Nebraska-Lincoln. We thank Christian Elowsky of the Morrison Microscopy Core Research Facility, University of Nebraska-Lincoln, for technical support.

\section{Author contributions}

R.A.W. designed the experiments. J.F., K.T.Y., K.M.C., J.D.W. and R.A.W. performed the experiments. R.A.W. interpreted the results. R.A.W. wrote the paper.

\section{Additional information}

Supplementary information accompanies this paper at http://www.nature.com/ scientificreports

Competing financial interests: The authors declare no competing financial interests.

How to cite this article: Fernandez, J., Yang, K.T., Cornwell, K.M., Wright, J.D. \& Wilson, R.A. Growth in rice cells requires de novo purine biosynthesis by the blast fungus Magnaporthe oryzae. Sci. Rep. 3, 2398; DOI:10.1038/srep02398 (2013).

This work is licensed under a Creative Commons AttributionNonCommercial-NoDerivs 3.0 Unported license. To view a copy of this license, visit http://creativecommons.org/licenses/by-nc-nd/3.0 